\title{
Nonlinear particle reacceleration by successive shocks
}

\author{
Thibault Vieu, ${ }^{a, *}$ Stefano Gabici $^{a}$ and Vincent Tatischeff ${ }^{b}$ \\ ${ }^{a}$ Université de Paris, CNRS, Astroparticule et Cosmologie, \\ F-75013 Paris, France \\ ${ }^{b}$ Université Paris-Saclay, CNRS/IN2P3, IJCLab, \\ 91405 Orsay, France \\ E-mail: vieu@apc.in2p3.fr
}

Multiple shock acceleration is thought to take place in various environments, including chaotic winds, accretion disks and superbubbles. In the latter, multiple supernovae may explode within small timescales in compact regions and successively reaccelerate the distribution of cosmic rays which do not have time to escape far away from the star cluster. Reacceleration by successive shock waves is therefore expected to take place. We develop a model of nonlinear shock reacceleration and show that the downstream distribution of particles rapidly reach an asymptotic concave solution, such that the cosmic ray pressure saturates at the level of a few percent of the hydrodynamic pressure.

$37^{\text {th }}$ International Cosmic Ray Conference (ICRC 2021)

July 12 th - 23rd, 2021

Online - Berlin, Germany

\footnotetext{
${ }^{*}$ Presenter
} 


\section{Introduction}

The problem of particle reacceleration by successive shocks has been early tackled in the testparticle regime [1-4]. It was shown that the spectrum hardens until it reaches an asymptotic $p^{-3}$ power-law. However, the pressure of the reaccelerated particles is expected to quickly rise above the ram pressure of the shocks, which implies that the backreaction of the particles onto the shocks must be taken into account. While many models have been devoted to the modelling of cosmic ray acceleration by nonlinear shocks (e.g. [5-9]), there have been very few attempts to account for the presence of preexisting "seed" particles [10-12]. We therefore seek to develop an up-to-date semi-analytical modelling in order to compute the reacceleration of particles by many successive shocks.

\section{Nonlinear model of shock reacceleration}

\subsection{Kinetic equation}

We consider a plane infinite shock propagating along the $x$ axis with a flow profile $u(x)$. The distribution of seed particles upstream of the shock is denoted $f_{\infty}$ while the total distribution of particles, including the reaccelerated seeds as well as the particles accelerated from the thermal bath, is denoted $f$. The transport equation integrated around the shock reads [7]:

$$
\frac{p}{3}\left(u_{2}-u_{p}\right) \frac{\mathrm{d} f_{1}}{\mathrm{~d} p}=\left(u_{p}+\frac{p}{3} \frac{\mathrm{d} u_{p}}{\mathrm{~d} p}\right) f_{1}-u_{0} f_{\infty}-Q_{1} \delta(x) \delta\left(p-p_{0}\right),
$$

with $u_{p}$ defined as:

$$
u_{p}(p) \equiv u_{1}-v_{A, 1}-\frac{1}{f_{1}(p)} \int_{-\infty}^{0} \mathrm{~d} x \mathrm{~d}_{x}\left(u-v_{A}\right) f(x, p) .
$$

The indices " 0 ", " 1 " and " 2 " respectively denote quantities evaluated at upstream infinity, immediately upstream of the shock and immediately downstream of the shock. $v_{A}$ is the Alfvén velocity which is introduced in order to account for the Alfvénic drift [13]. $Q_{1}$ is the injection rate of particles from the thermal bath, determined such that the non-thermal distribution $f$ matches the thermal (Maxwell Boltzmann) distribution at the injection momentum, which eventually provides the following boundary condition [14]:

$$
f_{1}\left(p_{0}\right)=\frac{n_{0} R_{t o t}}{\pi^{3 / 2} p_{0}^{3}} \xi^{3} e^{-\xi^{2}},
$$

where $n_{0}$ is the density of the plasma at upstream infinity, $R_{t o t}=u_{0} / u_{2}, p_{0}$ is the injection momentum and $\xi \approx 2.5-4$ the injection parameter.

\subsection{Fluid equation}

The hydrodynamic profile of the shock is driven by the conservation of mass and momentum. The latter reads:

$$
1+P_{g, 0}+P_{c, 0}=U(x)+P_{g}(x)+P_{c}(x)+P_{B}(x),
$$


where the pressures have been normalised with respect to the ram pressure $\rho_{0} u_{0}^{2}$ and $U=u / u_{0}$. $P_{c}(x)=4 \pi /\left(3 \rho_{0} u_{0}^{2}\right) \int_{p_{0}}^{\infty} \mathrm{d} p p^{3} v(p) f(x, p)$ is the pressure of the particles. The gas pressure $P_{g}$ is obtained assuming an adiabatic equation of state of index $\gamma(=5 / 3): P_{g}(x)=U^{-\gamma}(x) /\left(\gamma M_{0}^{2}\right)$. The magnetic pressure $p_{B}$ is obtained by solving the transport equation of the magnetic field amplified by resonant streaming instability, which provides $P_{B}(x)=(2 / 25)\left(1-U(x)^{5 / 4}\right)^{2} U(x)^{-3 / 2}[13]$.

\subsection{Method of resolution}

An approximate solution of the problem is obtained by introducing the typical distance $x_{p}(p)$ probed by particles of momentum $p$ ahead of the shock [10,15]. The spatial dependency of the distribution function can be approximately explicated as:

$$
f(x, p)=\left[f_{1}(p)-f_{\infty}(p)\right] \vartheta\left[x-x_{p}(p)\right]+f_{\infty}(p),
$$

such that the expression of the cosmic ray pressure simplifies to:

$$
P_{c}\left(x_{p}\right) \approx \frac{4 \pi}{3 \rho_{0} u_{0}^{2}}\left\{\int_{p_{0}}^{p} \mathrm{~d} p^{\prime} p^{\prime 3} v\left(p^{\prime}\right) f_{\infty}\left(p^{\prime}\right)+\int_{p}^{\infty} \mathrm{d} p^{\prime} p^{\prime 3} v\left(p^{\prime}\right) f_{1}\left(p^{\prime}\right)\right\} .
$$

Defining $\zeta(p) \equiv U\left(x_{p}(p)\right)$, the problem reduces to a system of coupled ordinary differential equations:

$$
\begin{aligned}
& \frac{p}{3} \frac{\mathrm{d} f_{1}}{\mathrm{~d} p}\left(\frac{1}{R_{t o t}}-\frac{7}{5} \zeta+\frac{2}{5} \zeta^{-1 / 4}\right)=\frac{f_{1}-f_{\infty}}{5}\left(7 \zeta-2 \zeta^{-1 / 4}+\frac{p}{6}\left(14+\zeta^{-5 / 4}\right) \zeta^{\prime}(p)\right), \\
& \zeta^{\prime}(p)\left[\frac{27}{25}-\frac{\zeta^{-\gamma-1}}{M_{0}^{2}}+\frac{\zeta^{-5 / 4}}{25}-\frac{3 \zeta^{-5 / 2}}{25}\right]=\frac{4 \pi}{3 \rho_{0} u_{0}^{2}} p^{3} v(p)\left[f_{1}(p)-f_{\infty}(p)\right]
\end{aligned}
$$

which can be solved iteratively with suitable boundary conditions determined by the RankineHugoniot jump conditions at the subshock [8]. The initial value of the distribution function $f$ is given by Equation 3 while the hydrodynamic equation 8 is supplemented by the boundary condition $\zeta\left(p_{\max }\right)=u_{0}$, for the flow should not be modified far upstream. Because nonlinear shocks generally produce hard spectra in the high energy bands, it is mandatory to introduce a maximum momentum $p_{\max }$ which is equivalent to assume a free escape boundary in the upstream region. The solution is eventually found following the procedure detailed in [10].

\subsection{Adiabatic decompression and escape flux}

The medium is compressed by a factor $u_{0} / u_{2}$ during the passage of a shock. In between two shocks, the decompression must be taken into account. This is properly computed according to Liouville's theorem as discussed in [4].

Because we introduce a maximum momentum $p_{\max }$, a non-negligible fraction of particles may escape at upstream infinity. The escape flux is computed as in [16], including the additional pressure of the seeds at upstream infinity. 

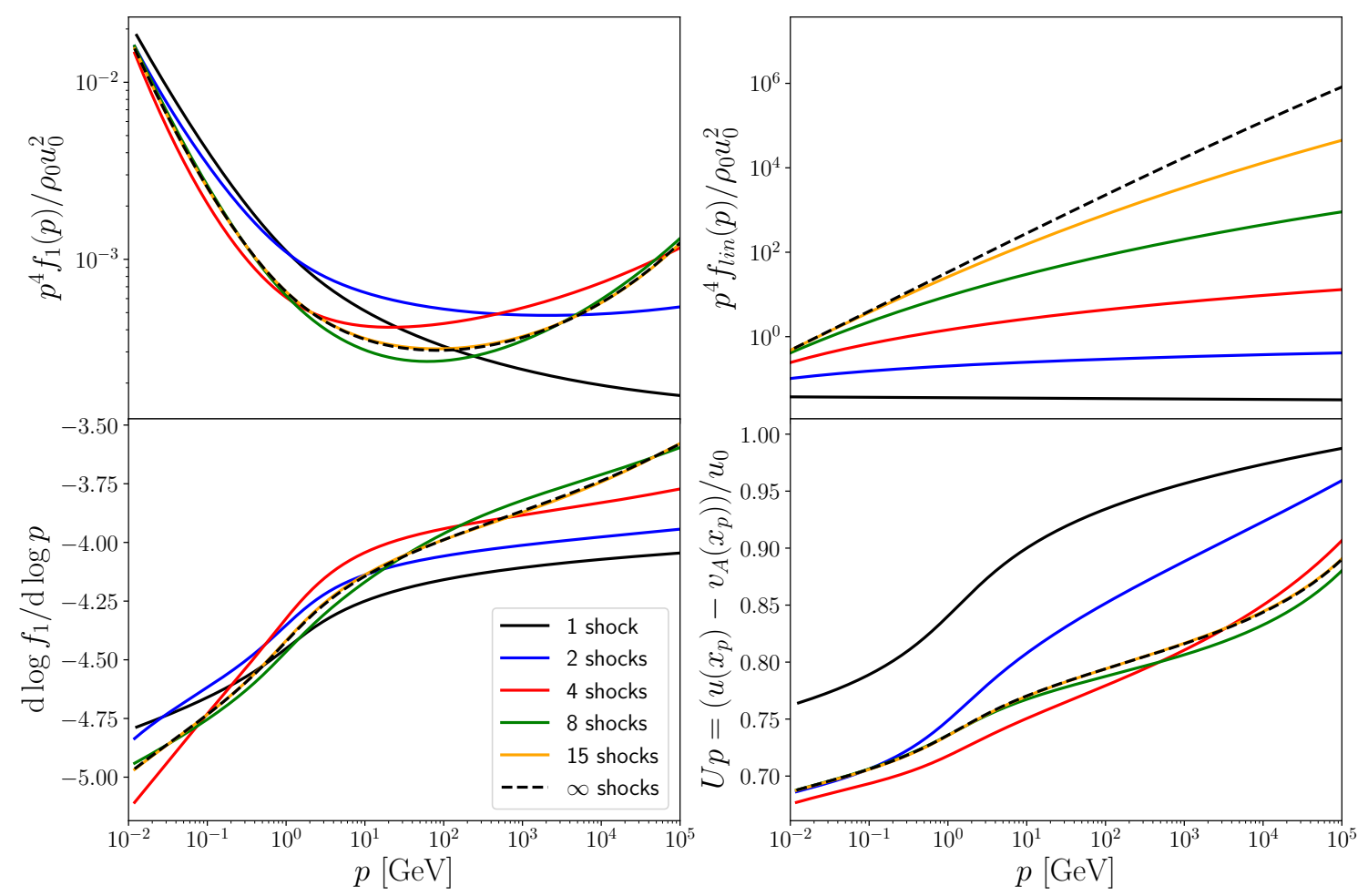

Figure 1: Evolution of the downstream distribution of particles (left panels) as the shocks succeed each other, compared to the test-particle solution (top right panel). The bottom right panel displays the profile of the flow effectively experienced by particles of momentum $p$. The Mach number is set to 20, the upstream temperature to $10^{6} \mathrm{~K}$, the injection parameter $\xi=3$ and the maximum momentum $p_{\max }=1 \mathrm{PeV}$.

\section{Results}

\subsection{Asymptotic solution}

Figure 1 shows the evolution of the cosmic ray spectrum and flow profile as the shocks successively sweep-up the medium. It is well-known that the pressure of cosmic rays slows down the flow ahead of the shock, as shown in the bottom right panel. As the shocks succeed each other, the pressure of the particles increases and the flow is more and more modified. This implies that low energy particles feel a reduced compression ratio which makes their acceleration inefficient, while high energy particles feel an enhanced compression ratio and are very efficiently (re)accelerated. This results in a more and more concave spectrum, in contrast to the test-particle case (top right panel of Figure 1). The asymptotic solution has a spectral index of about 5 at the injection momentum and 3.5 at the maximum momentum.

Remarkably, the asymptotic solution is nearly universal. As shown in Figure 2, it does not depend on the Mach number, even for weak shocks. Neither does it depend on the maximum momentum, providing the spectrum is rescaled accordingly. Only low energy bands do depend on the injection efficiency, which is expected since the fresh injection of particles is introduced as a boundary condition.

Figure 3 shows how the pressure of the downstream distribution of cosmic rays evolves as the 

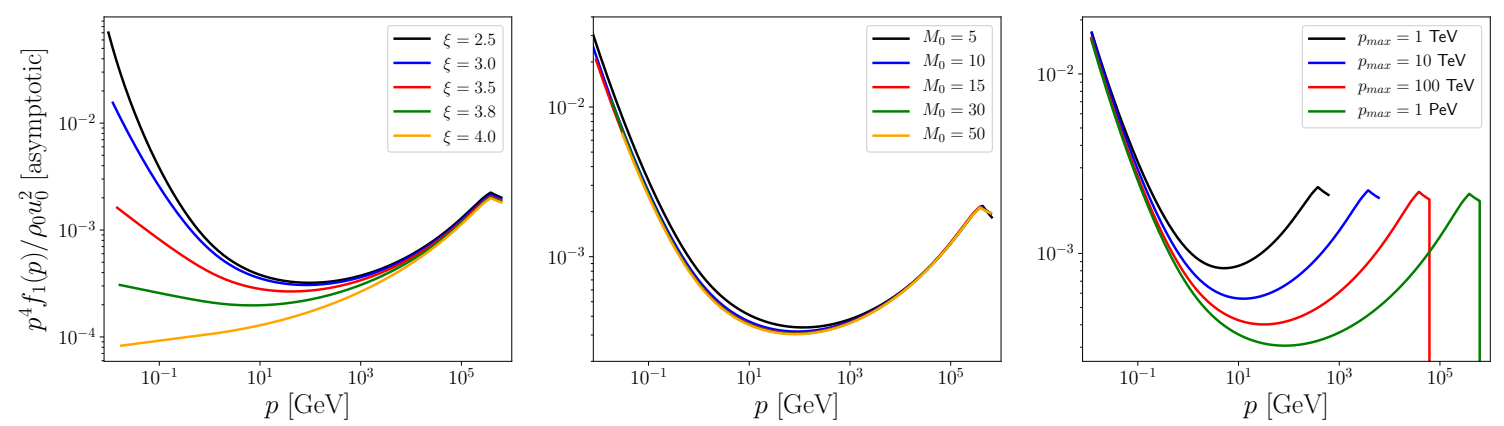

Figure 2: Asymptotic solutions for various sets of parameters. Left: the Mach number is set to 20, the maximum momentum to $1 \mathrm{PeV}$. Middle: $\xi=3, p_{\max }=1 \mathrm{PeV}$. Right: the Mach number is set to $20, \xi=3$.
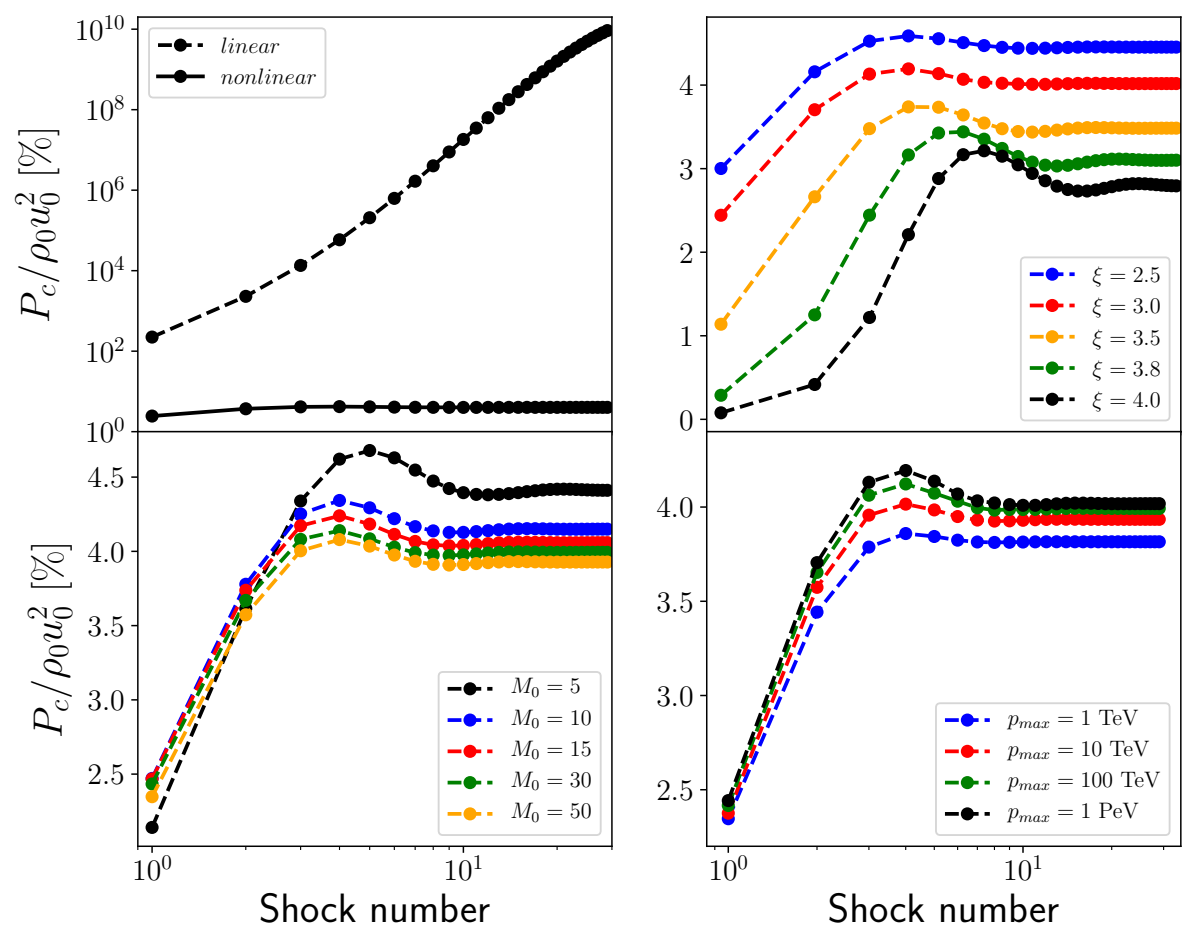

Figure 3: Evolution of the cosmic ray pressure in the downstream region. The parameters are similar to that set in Figure 2.

shocks succeed each other. The top left panel demonstrates that nonlinearities must be taken into account even when considering a small number of shocks. The pressure asymptotically converges to about a few percent of the shock ram pressure, for any set of parameters. In particular, even assuming a very small acceleration efficiency (e.g. $10^{-10}$ percent of thermal particles injected in the mechanism), the pressure saturates after about 10 reaccelerations at the level of a few percent of the hydrodynamic pressure. In the saturated regime, only a fraction of the seeds are reaccelerated and most of the energy is carried by the escape flux at the maximum momentum. 


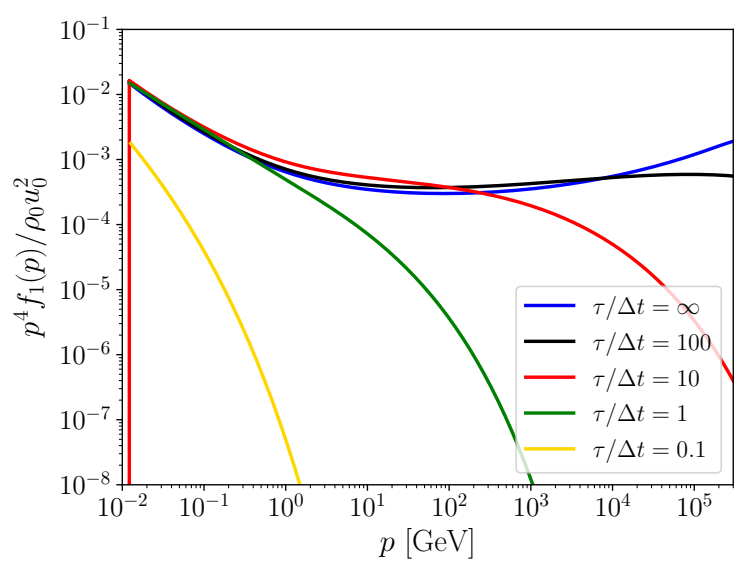

Figure 4: Asymptotic spectrum accounting for the expansion of the medium and particle escape. We assume a Mach number of 20 and $\xi=3$.

\subsection{Particle escape}

The previous computation was performed in an idealistic scenario where the properties of the swept-up medium are constant in time and the particles do not escape the region of acceleration. In reality, the medium must be heated by the successive shocks, unless it expands. A more realistic model can be implemented by accounting for an adiabatic expansion of the region, which is typically the case in superbubble environments. We assume that the density decreases in between the passage of each shock according to Weaver's scaling [17] and we allow for the escape of particles in a timescale $\tau(p) \propto p^{-1 / 3}$ as expected in the Kolmogorov regime of turbulence [18]. Figure 4 shows how the asymptotic solution is modified. Although the rarefaction of the medium does not have a major impact on the spectrum of particles, the escape of cosmic rays steepens the high energy bands. If the escape time of low energy particles is smaller than the typical time interval between supernovae $\Delta t$, the spectrum is very steep. The asymptotic solution is in fact only approximatively retrieved up to the momentum $p_{*}$ such that $\tau\left(p_{*}\right)=\Delta t$. The concave spectrum from the injection momentum to $1 \mathrm{PeV}$ would only be realised in very massive compact clusters containing more than a thousand massive stars, such as the ones observed in the galactic centre.

\section{Conclusion}

Successive shocks efficiently accelerate cosmic rays such that the pressure of the particles quickly raises at the level of the ram pressure of the shocks. This implies that the test-particle approximation breaks down. The cosmic rays react onto the shock and in particular slow down the upstream flow. We have computed the reacceleration of particles in such setup using a semianalytical model including the resonant excitation of hydromagnetic waves and self-consistently accounting for the injection of particles. The asymptotic spectrum obtained after a few reaccelerations is very concave. In the nonlinear regime the cosmic ray saturates at the level of a few percent of the shock ram pressure and most of the energy is carried by the escaping flux. Remarkably, the asymptotic solution is nearly universal. In particular, the asymptotic pressure weakly depends on the injection efficiency. The model is eventually applied to a more realistic case considering 
the expansion of the medium as well as the energy-dependent escape of the particles, which is a step towards a nonlinear model of acceleration in superbubbles. The asymptotic spectrum is only retrieved if the shocks succeed each other very quickly. Eventually, the framework developed in this analysis can be used to compute the reacceleration of seed particles in various environments, not limited to superbubbles.

\section{References}

[1] A.R. Bell, The acceleration of cosmic rays in shock fronts - II., MNRAS 182 (1978) 443.

[2] R.L. White, Synchrotron emission from chaotic stellar winds., ApJ 289 (1985) 698.

[3] A. Achterberg, Particle acceleration by an ensemble of shocks, A\&A 231 (1990) 251.

[4] D.B. Melrose and M.H. Pope, Diffusive Shock Acceleration by Multiple Shocks, Proceedings of the Astronomical Society of Australia 10 (1993) 222.

[5] L.O. Drury and J.H. Voelk, Hydromagnetic shock structure in the presence of cosmic rays, ApJ 248 (1981) 344.

[6] M.A. Malkov and H.J. Voelk, Renormalized Two-Fluid Hydrodynamics of Cosmic-Ray-modified Shocks, ApJ 473 (1996) 347.

[7] P. Blasi, A semi-analytical approach to non-linear shock acceleration, Astroparticle Physics 16 (2002) 429 [astro-ph/0104064].

[8] D. Caprioli, P. Blasi, E. Amato and M. Vietri, Dynamical feedback of self-generated magnetic fields in cosmic ray modified shocks, MNRAS 395 (2009) 895 [0807.4261].

[9] D. Caprioli and A. Spitkovsky, Simulations of Ion Acceleration at Non-relativistic Shocks. I. Acceleration Efficiency, ApJ 783 (2014) 91 [1310.2943].

[10] P. Blasi, Nonlinear shock acceleration in the presence of seed particles, Astroparticle Physics 21 (2004) 45.

[11] H. Kang and D. Ryu, Re-acceleration of Non-thermal Particles at Weak Cosmological Shock Waves, ApJ 734 (2011) 18 [1102.2561].

[12] D. Caprioli, H. Zhang and A. Spitkovsky, Diffusive shock re-acceleration, Journal of Plasma Physics 84 (2018) 715840301 [1801.01510].

[13] D. Caprioli, Cosmic-ray acceleration in supernova remnants: non-linear theory revised, J. Cosmology Astropart. Phys. 2012 (2012) 038 [1206. 1360].

[14] P. Blasi, S. Gabici and G. Vannoni, On the role of injection in kinetic approaches to non-linear particle acceleration at non-relativistic shock waves, MNRAS 361 (2005) 907-918. 
[15] D. Eichler, Particle acceleration in collisionless shocks: regulated injection and high efficiency., ApJ 229 (1979) 419.

[16] D. Caprioli, P. Blasi and E. Amato, On the escape of particles from cosmic ray modified shocks, Monthly Notices of the Royal Astronomical Society 396 (2009) 2065.

[17] R. Weaver, R. McCray, J. Castor, P. Shapiro and R. Moore, Interstellar bubbles. II. Structure and evolution., ApJ 218 (1977) 377.

[18] G. Ferrand and A. Marcowith, On the shape of the spectrum of cosmic rays accelerated inside superbubbles, A\&A 510 (2010) A101 [0911.4457]. 